\title{
Genome-Wide Association Study of Medication Adherence in Chronic Diseases in the Korean Population
}

\author{
Incheol Seo, Seong-Il Suh, Min-Ho Suh, Won-Ki Baek* \\ Department of Microbiology, Keimyung University School of Medicine, Daegu 704-701, Korea
}

\begin{abstract}
Medication adherence is generally defined as the extent of voluntary cooperation of a patient in taking medicine as prescribed. Adherence to long-term treatment with chronic disease is essential for reducing disease comorbidity and mortality. However, medication non-adherence in chronic disease averages $50 \%$. This study was conducted a genome-wide association study to identify the genetic basis of medication adherence. A total of 235 medication non-adherents and 1,067 medication adherents with hypertension or diabetes were used from the Korean Association Resource project data according to the self-reported treatment status of each chronic disease, respectively. We identified four single nucleotide polymorphisms with suggestive genome-wide association. The most significant single nucleotide polymorphism was rs6978712 (chromosome 7, $\mathrm{p}=4.87 \times 10^{-7}$ ), which is located proximal to the GCC1 gene, which was previously implicated in decision-making capability in drug abusers. Two suggestive single nucleotide polymorphisms were in strong linkage disequilibrium $\left(r^{2}>0.8\right)$ with $r 6978712$. Thus, in the aspect of decision-making in adherence behavior, the association between medication adherence and three loci proximal to the GCC1 gene seems worthy of further research. However, to overcome a few limitations in this study, defining the standardized phenotype criteria for self-reported adherence should be performed before replicating association studies.
\end{abstract}

Keywords: chronic disease, genome-wide association study, medication adherence, medication compliance

\section{Introduction}

Medication adherence is generally defined as the extent to which patient take medications to coincide with the healthcare provider's recommendation [1]. Adherence to long-term treatment in chronic disease, such as hypertension and diabetes, is essential for reducing the risks of disease comorbidity and mortality [2]. Poor adherence to medication can cause worsening of disease, death, and increased financial burden for society [1]. Most cases of hypertension and diabetes are inextirpable. The main goal of treatment for these chronic diseases is to delay disease progression and to prevent developing complications. Thus, medication adherence is one of the most important factors for success in lifelong treatment [3]. However, medication non-adherence in chronic disease averages 50\% [4]. The World Health Organi- zation reported that non-adherence is a leading cause of preventable death and cost. Predictors of poor adherence are a useful indicator for physicians to identify patients who are most in need of interventions to improve adherence [1]. Illness perceptions and self-efficacy are powerful predictors of adherence [2]. These health-related cognitions have associations with personality, and personality traits can be conveniently summarized by a five-factor model of personality (conscientiousness, neuroticism, agreeableness, extraversion, and openness) [5]. Several studies have explored the associations between personality and adherence. Among the 12 previous publications exploring associations between personality traits and medication adherence, 7 studies reported that medication adherence has been positively related to conscientiousness, 5 studies reported negative relationships with neuroticism, 2 studies reported about agreeableness with positive associations, and 1 study reported a 
positive relationship with extraversion [6-8]. A genetic basis of personality traits has been identified from behavior genetic studies. Twin, adoption, and family studies show that heritability of personality is about 50\% [9]. Several studies have attempted to identify candidate genes with five dimensions of personality, but the results were inconclusive. Genetic linkage studies have suggested only a few genomic regions for personality traits. The genetic influences on personality traits are likely attributable to many genes with small effects. Few genome-wide association studies (GWASs) have identified genetic variants associated with personality traits. However, most of the associations with personality traits could not be replicated, except in one study [8]. Unlike the attempts to elucidate the genomic associations of personality, there is no research on the genetic aspect of medication adherence until now. We assumed that medication adherence has a genetic background, as the personality traits that affect medication adherence have a genetic basis for heritability. Therefore, we conducted a GWAS to identify the association between medication adherence and genotype.

\section{Methods}

\section{Study subjects}

A nested case-control study of medication adherence in chronic diseases (hypertension or diabetes) was conducted within the Korean Association Resource (KARE) project data (which included genotypes from 8,842 participants from the rural Anseong and urban Ansan cohorts as part of the Korean Genome Epidemiology Study) [10]. Two groups were extracted from the KARE data, one of medication non-adherents with chronic disease (cases) and the other of medication adherents with chronic disease (controls), based on the following procedure (Fig. 1). There were 1,387 and 613 individuals who were diagnosed with hypertension and diabetes, respectively, of a total of 8,842; 1,540 individuals were defined as patients with chronic disease after excluding 230 patients with comorbid hypertension and diabetes. Among 1,540 patients with chronic disease, a patient who was undergoing treatment for hypertension or diabetes or who was taking hypertensive or diabetes medicines (including insulin) were classified as the control group $(\mathrm{n}=$ 1,072). Patients who was not receiving treatment or taking medicines were classified as the case group $(n=239)$. Individuals whose information about treatment was not provided were removed $(n=229)$. Consequently, 235 cases and 1,067 controls were used for the GWAS after excluding 9 patients with comorbid psychotic disorders or cognitive disorders, such as dementia [1].

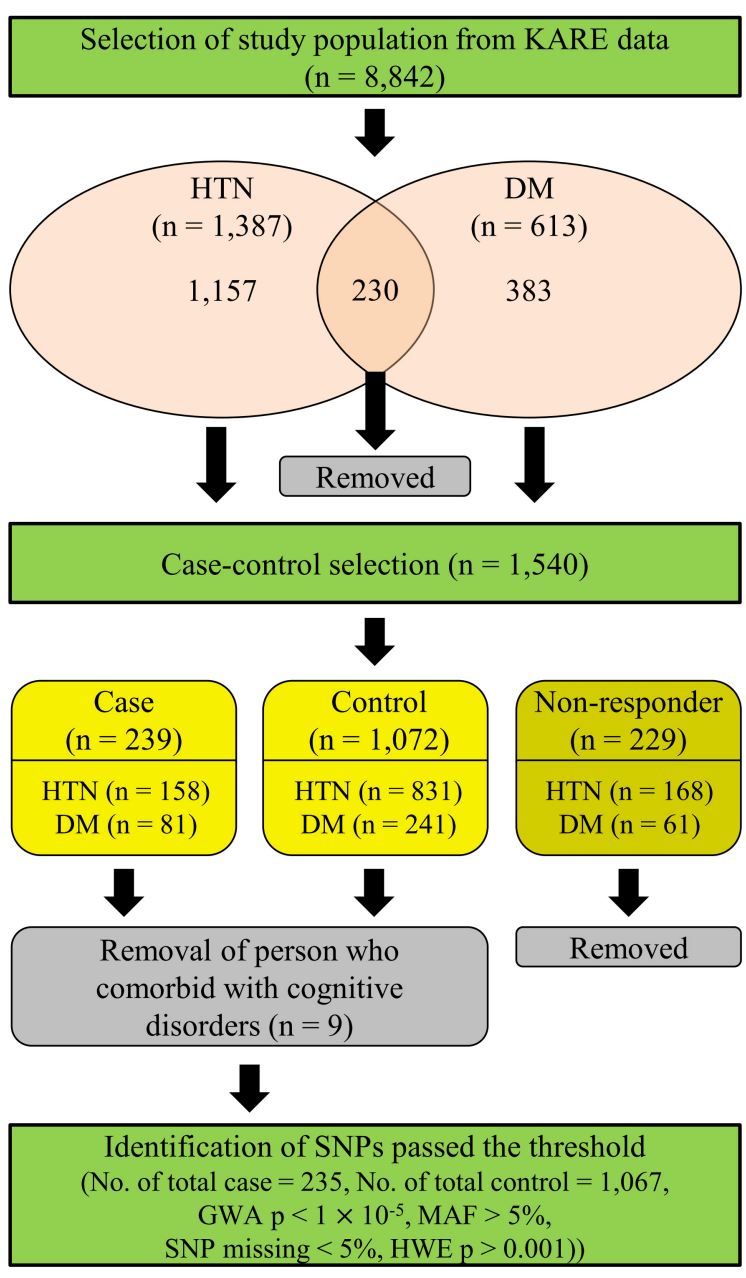

Fig. 1. Summary of the sampling and quality control procedure. KARE, Korean Association Resource project; HTN, hypertensive patients; DM, diabetes patients; SNP, single nucleotide polymorphism; GWA, genome-wide association; MAF, minor allelic frequency; HWE, Hardy-Weinberg equilibrium.

\section{Genotyping}

The genotyping and quality control procedures for the KARE data have been described previously [10]. Single nucleotide polymorphisms (SNPs) with a high missing gene call rate $(>5 \%)$, low minor allele frequency $(<0.05)$, and significant deviation from Hardy-Weinberg equilibrium $(\mathrm{p}$ $\leq 0.001$ ) were excluded, leaving a total of 311,493 SNPs to be examined.

\section{Statistical analyses}

The case-control GWAS was conducted using PLINK software version 1.07 [11]. A quantile-quantile (QQ) plot and a Manhattan plot were produced using the R statistics package [12]. A regional plot was generated using LocusZoom 1.1 [13]. Student's t-test and Fisher's exact test were used to test differences in basic and clinical characteristics between 
Table 1. Characteristics of the study subjects

\begin{tabular}{lccc}
\hline \multicolumn{1}{c}{ Variable } & Case (non-adherents, $\mathbf{n}=\mathbf{2 3 5}$ ) & Control (adherents, $\mathbf{n}=\mathbf{1 , 0 6 7}$ ) & p-value \\
\hline DM/HTN (no. of persons) & $80 / 155$ & $240 / 827$ & $0.0003^{\mathrm{a}}$ \\
Gender (male/female) & $116 / 119$ & $440 / 627$ & $0.024^{\mathrm{a}}$ \\
Mean age (y) & $54.29 \pm 8.10$ & $57.66 \pm 7.90$ & $<0.0001^{\mathrm{b}}$ \\
Blood pressure in hypertensive patients & & & \\
Systolic blood pressure (mm Hg) & $141.56 \pm 19.13$ & $138.43 \pm 18.23$ & $0.0518^{\mathrm{b}}$ \\
Diastolic blood pressure (mm Hg) & $90.99 \pm 11.46$ & $88.64 \pm 10.59$ & $0.0125^{\mathrm{b}}$ \\
FPG in diabetic patients (mg/dL) & $135.20 \pm 60.35$ & $142.31 \pm 50.81$ & $0.3857^{\mathrm{b}}$ \\
FHX of HTN in hypertensive patients (yes/no) & $54 / 101$ & $296 / 531$ & $0.8553^{\mathrm{a}}$ \\
FHX of DM in diabetic patients (yes/no) & $22 / 58$ & $63 / 177$ & $0.8839^{\mathrm{a}}$ \\
FHX of CVA (yes/no) & $38 / 197$ & $144 / 923$ & $0.2988^{\mathrm{a}}$ \\
FHX of CAD (yes/no) & $2 / 233$ & $31 / 1,064$ & $0.1024^{\mathrm{a}}$ \\
\hline
\end{tabular}

Values are presented as mean $\pm \mathrm{SD}$.

DM, diabetes mellitus; HTN, hypertension; FPG, fasting plasma glucose; FHX, presence of family history; CVA, cerebrovascular attack; CAD, coronary artery disease; SD, standard deviation.

p-values were derived using either ${ }^{\mathrm{a}}$ Fisher's exact test (two-tailed); ${ }^{\mathrm{b}}$ Student's t-test (two-tailed); ${ }^{\mathrm{C}} 71$ cases and 119 controls were compared after excluding individuals with missing values.
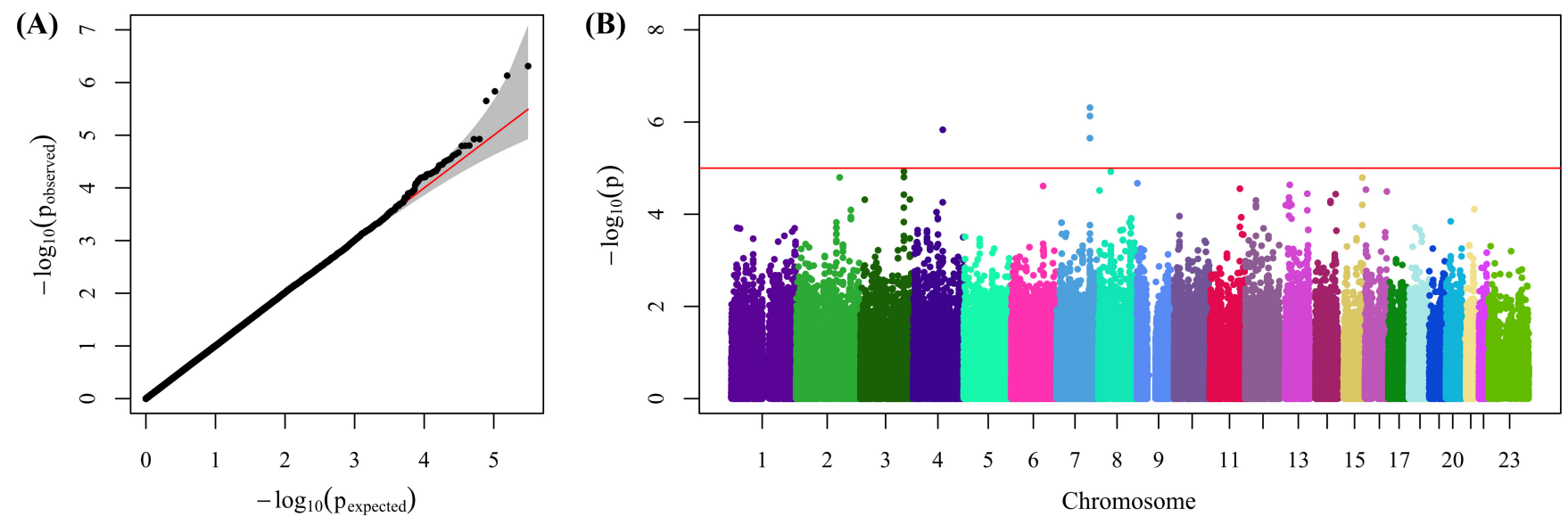

Fig. 2. Quantile-quantile (QQ) plot and Manhattan plot of genome-wide association study (GWAS) for medication adherence. (A) QQ plot shows the differences between the observed and expected $-\log _{10}$ ( $\mathrm{p}$-value) of the study results. The red line indicates the expected distributions under the null hypothesis. The gray shaded region represents the $95 \%$ confidence interval. (B) Manhattan plot of single nucleotide polymorphisms for medication adherence. X-axis represents chromosomes. Y-axis represents $-\log _{10}$ ( $p$-values) obtained by genome-wide association analysis. The red line indicates the suggestive threshold for genome-wide significance $\left(\mathrm{p}<1 \times 10^{-5}\right)$.

case and control groups.

\section{Results}

\section{Characteristics of study subjects}

The general and clinical characteristics of the case and control groups are described in Table 1. The case group (non-adherents) consisted of 80 subjects with diabetes and 155 individuals with hypertension, and the control group (adherents) consisted of 240 and 827, respectively. Individuals with diabetes tended to adhere less to medication than hypertensive patients [14]. The mean age of the case group was $54.29 \pm 8.10$ and $57.66 \pm 7.90$ years in the control group. Adherents were significantly older than non-adherents. Males were less adherent than females. All results above are consistent with previous studies of factors affecting medication adherence [3]. Among the clinical characteristics, diastolic blood pressure in hypertensive individuals was higher in the non-adherence group. However, systolic blood pressure in hypertensive patients and fasting plasma glucose in diabetic person were similar between adherence and non-adherence groups. The rates of family history of hypertension, diabetes, and their life-threatening complications, such as cerebrovascular attack and coronary artery disease, did not differ between case and control groups. 
Table 2. SNPs with $p<1 \times 10^{-5}$ in the GWAS of 235 medication non-adherent cases and 1,067 medication adherent controls from the KARE cohort

\begin{tabular}{|c|c|c|c|c|c|c|c|c|}
\hline rs ID & Chr & Nearest gene & Position & $\mathrm{A} 1 / \mathrm{A} 2$ & $\begin{array}{c}\text { MAF } \\
\text { in case. }\end{array}$ & $\begin{array}{c}\text { MAF } \\
\text { in cont. }\end{array}$ & OR $(95 \% \mathrm{Cl})$ & p-value \\
\hline rs6978712 & 7 & GCC1 & $126,934,999$ & $\mathrm{~A} / \mathrm{G}$ & 0.175 & 0.095 & $2.02(1.53-2.68)$ & 4.87E-07 \\
\hline rs10487488 & 7 & GCC1 & $126,936,527$ & $\mathrm{C} / \mathrm{A}$ & 0.175 & 0.096 & $2.00(1.51-2.64)$ & $7.40 \mathrm{E}-07$ \\
\hline rs7690819 & 4 & LYPLA1P2 & $112,340,107$ & $\mathrm{G} / \mathrm{A}$ & 0.521 & 0.4 & $1.63(1.34-2.00)$ & $1.47 \mathrm{E}-06$ \\
\hline rs11973012 & 7 & GCC1 & $126,960,898$ & $\mathrm{C} / \mathrm{T}$ & 0.175 & 0.098 & $1.94(1.47-2.56)$ & $2.24 \mathrm{E}-06$ \\
\hline
\end{tabular}

SNP, single nucleotide polymorphism; GWAS, genome-wide association study; KARE, Korean Association Resource; rs ID, reference SNP ID obtained from dbSNP database; Chr, Chromosome; A1/A2, minor/major allele; MAF in case., minor allele frequencies in cases; MAF in cont., minor allele frequencies in controls; $\mathrm{OR}$, odds ratio; $\mathrm{Cl}$, confidence interval.

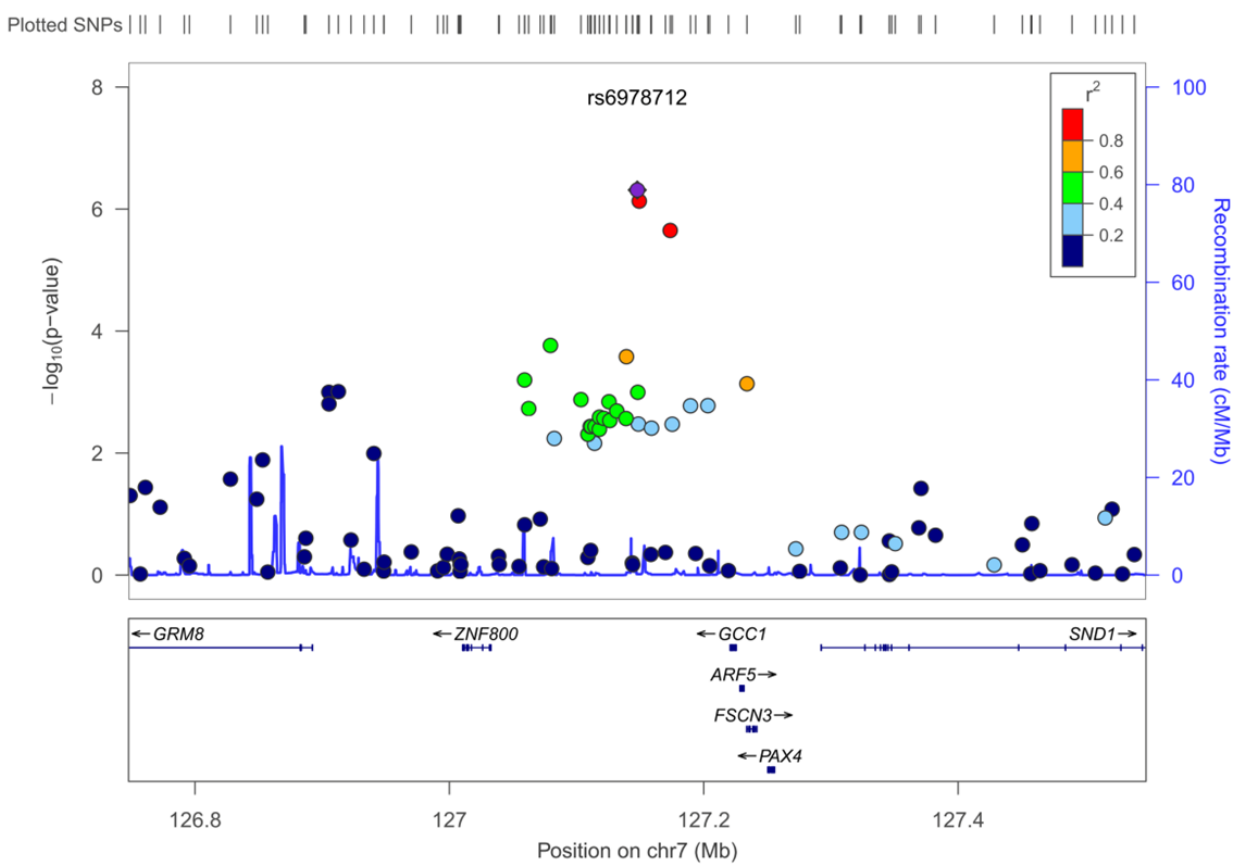

Fig. 3. Regional association plot of the most significant loci associated with medication adherence. Purple circle indicates the strongest association (rs6978712) detected in the genomewide analysis. Other circles indicate SNPs genotyped in this study, and their color reflects the linkage disequilibrium $\left(r^{2}\right)$ with rs6978712. The blue line represents the recombination rates in the 1000 Genome Mar 2012 ASN population. SNPs, single nucleotide polymorphisms; ASN, super population of the east Asian, which includes Han Chinese in Beijing, China; Japanese in Tokyo, Japan; Southern Han Chinese; Chinese Dai in Xishuangbanna, China; and Kinh in Ho Chi Minh City, Vietnam.

\section{SNPs associated with medication adherence}

The GWAS for medication adherence in 1,067 medication adherents and 235 medication non-adherents showed multiple SNPs with a suggestive association (Fig. 2). The QQ plot showed that most of the observed associations lay close to the expected distribution, conforming to the null hypothesis of no association. The genomic inflation factor was 1.00, indicating no evidence of inflation in the test statistics due to population stratification or other sources. SNPs with a suggestive genome-wide association $\left(\mathrm{p}<1 \times 10^{-5}\right)$ are shown in Table 2. Although no SNP met the criteria of genome-wide significance $\left(\mathrm{p}<5 \times 10^{-8}\right.$ ), four loci (rs6978712, rs10487488, rs7690819, rs11973012) with suggestive significance were identified. The most significant SNP was rs6978712 ( $\mathrm{p}=$ $4.87 \times 10^{-7}$ ), which is located proximal to the $G C C 1$ gene, which was previously implicated in decision-making capa- bility in drug abusers [15]. Three SNPs on chromosome 7 were in close proximity, and Fig. 3 shows a regional association for these SNPs proximal to the $G C C 1$ gene.

\section{Discussion}

Medication adherence averages only $50 \%$ among patients receiving treatment for chronic diseases. Poor medication adherence contributes to worsening disease, death, and increased health care costs. Moreover, if medication adherence is not guaranteed, new therapeutic options, such as personalized medicine that aims to customize healthcare to individual patients based on their genetic background, are merely useless [16]. However, in spite of its importance in personal and social aspects, the genetic background for medication adherence has never been studied. Since personality traits that have a genetic background affect behaviors, in- 
cluding medication non-adherence, this study aimed to explore the genetic basis of medication adherence in chronic diseases for the first time.

Among the hypertensive or diabetic patients from the KARE cohort study, 235 medication non-adherent individuals (case) and 1,067 medication adherent individuals (control) were extracted according to the self-reported treatment status for the disease from the cohort questionnaire. Patient self-reports are considered a simple and effective method to measure adherence, since a gold standard does not exist [1]. Patients with cognitive impairments that can affect adherence were excluded. Defining rigorous phenotype criteria is paramount to the success of a case-control GWAS [17]. Thus, the method of classifying individuals as either case or control in this study was validated by comparing the general characteristics of the case-control subjects. The differences in demographic factors (age and gender) and disease factors for adherence between the two groups were consistent with previous reports (Table 1) [3].

Nevertheless, the classification criteria in this study have limitations. First, there is the possibility of misclassification of treatment-adherent individuals as medication non-adherent patients, because the criteria were based exclusively on current medication status for hypertension or diabetes. For example, patients with mild hypertension or diabetes who follow instructions from the physician to modify their diet or lifestyle as an initial therapy can be classified as medication non-adherent individuals. Second, the different ratio of the number of hypertensive and diabetic patients between the case and control groups can affect the GWAS results [18]. Additionally, the risk of volunteer bias is present, in which individuals who participated in the KARE cohort possibly tended to adhere more to health care services. To overcome these limitations, standardized phenotype criteria for self-reported adherence, such as the five-item Medication Adherence Report Scale-MARS-5, which has already been used in various settings [19], and the eight-item Morisky Medication Adherence Scale-MMAS-8, which has been validated in over 1,000 individuals with hypertension [20], can be applied in the study design. These criteria enable reliable and replicable classification of medication adherence [17].

This study identified four loci that were suggestively associated with medication adherence. The most significantly associated SNP was rs6978712 $\left(\mathrm{p}=4.87 \times 10^{-7}\right)$, which is located nearby the $G C C 1$ gene on chromosome 7. Two suggestive SNPs were in strong linkage disequilibrium $\left(\mathrm{r}^{2}>0.8\right)$ with rs6978712 (Fig. 3). This might be due to the close physical proximity of those three SNPs. GCC1 encodes a peripheral membrane protein that may have a role in the organization of the trans-Golgi network subcompartment that is associated with membrane transport [21]. The trans-Golgi network is a major secretory pathway that sorts and directs newly synthesized proteins to subcellular destinations, including neuronal synapses. Increased transcription of $G C C 1$ in the brain cortex of drug abusers has been reported in one study [15]. This study indicates that GCC1 is involved in decision-making capabilities in drug abusers. Patients with chronic diseases weigh the benefits from adherence with treatment against the constraints on their daily lives and the perceived risks of treatment, such as side effects, time, money, and effort involved, either consciously or subconsciously [3]. Thus, in the aspect of decision-making in adherence behavior, the association between medication adherence and the three SNPs proximal to the GCC1 gene identified in this study seems worthy of further research. The personality trait-associated SNPs were not associated with medication adherence in this study. Because of the multivariate association between personality traits and medication adherence, it seems difficult to apply the personality trait-associated SNPs to the medication adherence-associated SNPs directly.

This study suggests potential evidence for a genetic basis of medication adherence in chronic disease patients. A complete identification of the genetic influences for medication adherence appears feasible, because medication adherence is complexly influenced by not only personality traits but also many factors, including socio-economic factors, healthcare-related factors, and demographic factors [3]. Nevertheless, identifying genetic associations for poor medication adherence in chronic disease patients requires further research. It would be helpful for physicians to initiate personalized medication therapy by modifying treatment strategies, such as changing the route of administration, the complexity of treatment, and the degree of behavioral change required, for chronic disease patients with expected poor medication adherence from their genetic background.

\section{Acknowledgments}

This work was supported by grants from the Korea Centers for Disease Control and Prevention, Republic of Korea (4845-301, 4851-302, 4851-307).

\section{References}

1. Osterberg L, Blaschke T. Adherence to medication. $N$ Engl J Med 2005;353:487-497.

2. Zwikker HE, van den Bemt BJ, Vriezekolk JE, van den Ende $\mathrm{CH}$, van Dulmen S. Psychosocial predictors of non-adherence to chronic medication: systematic review of longitudinal studies. Patient Prefer Adherence 2014;8:519-563.

3. Jin J, Sklar GE, Min Sen Oh V, Chuen Li S. Factors affecting therapeutic compliance: A review from the patient's perspective. Ther Clin Risk Manag 2008;4:269-286. 
4. World Health Organization. Adherence to long-term therapies: evidence for action. Geneva: World Health Organization, 2003. Accessed 2014 May 1. Available from: http://www.who. int/chp/knowledge/publications/adherence_full_report.pdf.

5. McCrae RR, Costa PT Jr. Personality in Adulthood: A Five-Factor Theory Perspective. New York: Guilford Press, 2003.

6. Jerant A, Chapman B, Duberstein P, Robbins J, Franks P. Personality and medication non-adherence among older adults enrolled in a six-year trial. Br J Health Psychol 2011;16(Pt 1): 151-169.

7. Axelsson M, Brink E, Lundgren J, Lötvall J. The influence of personality traits on reported adherence to medication in individuals with chronic disease: an epidemiological study in West Sweden. PLoS One 2011;6:e18241.

8. Kim HN, Roh SJ, Sung YA, Chung HW, Lee JY, Cho J, et al. Genome-wide association study of the five-factor model of personality in young Korean women. J Hum Genet 2013;58: 667-674.

9. Terracciano A, Sanna S, Uda M, Deiana B, Usala G, Busonero $\mathrm{F}$, et al. Genome-wide association scan for five major dimensions of personality. Mol Psychiatry 2010;15:647-656.

10. Cho YS, Go MJ, Kim YJ, Heo JY, Oh JH, Ban HJ, et al. A large-scale genome-wide association study of Asian populations uncovers genetic factors influencing eight quantitative traits. Nat Genet 2009;41:527-534.

11. Purcell S, Neale B, Todd-Brown K, Thomas L, Ferreira MA, Bender D, et al. PLINK: a tool set for whole-genome association and population-based linkage analyses. Am J Hum Genet 2007;81:559-575.

12. Turner S. Qqman: Q-Q and manhattan plots for GWAS data.
The Comprehensive R Archive Network, 2014. Accessed 2014 May 1. Available from: http://cran.r-project.org/web/packages/qqman/.

13. Pruim RJ, Welch RP, Sanna S, Teslovich TM, Chines PS, Gliedt $\mathrm{TP}$, et al. LocusZoom: regional visualization of genome-wide association scan results. Bioinformatics 2010;26:2336-2337.

14. Briesacher BA, Andrade SE, Fouayzi H, Chan KA. Comparison of drug adherence rates among patients with seven different medical conditions. Pharmacotherapy 2008;28:437-443.

15. Lehrmann E, Colantuoni C, Deep-Soboslay A, Becker KG, Lowe R, Huestis MA, et al. Transcriptional changes common to human cocaine, cannabis and phencyclidine abuse. PLoS One 2006; 1:e114.

16. Malhotra AK, Zhang JP, Lencz T. Pharmacogenetics in psychiatry: translating research into clinical practice. Mol Psychiatry 2012;17:760-769.

17. Bush WS, Moore JH. Chapter 11: Genome-wide association studies. PLoS Comput Biol 2012;8:e1002822.

18. Pearson TA, Manolio TA. How to interpret a genome-wide association study. JAMA 2008;299:1335-1344.

19. Horne R, Weinman J. Self-regulation and self-management in asthma: exploring the role of illness perceptions and treatment beliefs in explaining non-adherence to preventer medication. Psychol Health 2002;17:17-32.

20. Morisky DE, Ang A, Krousel-Wood M, Ward HJ. Predictive validity of a medication adherence measure in an outpatient setting. J Clin Hypertens (Greenwich) 2008;10:348-354.

21. Kjer-Nielsen L, Teasdale RD, van Vliet C, Gleeson PA. A novel Golgi-localisation domain shared by a class of coiled-coil peripheral membrane proteins. Curr Biol 1999;9:385-388. 